\title{
PROPOSITION D'UN CADRE D'ANALYSE DES RELATIONS INTERGROUPES CONFLICTUELLES POTENTIELLEMENT VIOLENTES OU DISCRIMINATOIRES DANS LE SPECTACLE SPORTIF
}

\author{
Patrick Bouchet, Philippe Castel et Marie-Françoise Lacassagne ${ }^{1}$
}

Pour de nombreux acteurs politiques, le sport est l'occasion de refonder les liens humains à l'intersection du local et de l'international, du pays et de la planète, de la très haute compétition surmédiatisée et de la pratique anonyme, de l'individuel et du partagé, de l'identité singulière et des appartenances. Il s'ensuit une valorisation de cet objet culturel qui trouve un écho favorable dans la vie civile. En effet, au cours de la dernière décennie, de nombreux observateurs ont constaté une globalisation et une diversification du spectacle sportif, qu'il se déroule à l'intérieur des enceintes dédiées à ses manifestations ou dans les lieux consacrés à sa retransmission. Ce type d'événements a progressivement suscité un engouement mondial qui trouve en partie son explication dans le développement de dispositifs médiatiques soutenus par des investisseurs privés au travers des chaînes thématiques.

Les retransmissions mettent en scène plusieurs caractéristiques de la mondialisation comme l'abondance et l'instantanéité de l'information, la valorisation individuelle et financière..., qui s'opposent à des

\footnotetext{
1 Laboratoire SPMS, Université de Bourgogne
}

Recherches en communication, $\mathrm{n}^{\circ} 30$ (2008). 
caractéristiques plus traditionnelles comme l'incertitude du résultat et le don de soi, l'attachement territorial et la suprématie locale, le respect de l'adversaire et de l'arbitre..., et deviennent, de ce fait, de plus en plus attractives. L'essor des moyens de diffusion et de réception du spectacle sportif génère ainsi des transformations d'ordre économique, juridique, éthique et gestionnaire pour les parties prenantes impliquées. Aujourd'hui, ce nouveau marché qui avoisine des dizaines de milliards d'euros se manifeste de manière importante et inégale à fois dans les secteurs « privé » (médias, marques, industries), " public » (Europe, Etats, Régions, Villes) et «sportif » (Fédérations, Ligues, Clubs).

Cet engouement s'explique, par ailleurs, par l'augmentation de la participation des publics, à la fois dans les enceintes dédiées au spectacle sportif (stades, gymnases...), devant des écrans géants (places publiques, salles des fêtes, cafés...) ou dans la sphère privée (télévision, ordinateur, téléphonie mobile). A ce titre, ces espaces de réception constituent de plus en plus des théâtres « sociaux » au sein desquels les spectateurs jouent un rôle d'acteur des gradins au terrain, des écrans géants aux bars, du home cinéma aux pièces à vivre... Cette évolution des modes de réception du spectacle sportif a très certainement favorisé la diversification des publics traditionnels dont les centres d'intérêts ne se limitent plus à soutenir leur équipe ou leur joueur pour qu'il remporte la victoire. Il est maintenant courant d'observer des spectateurs ou amateurs de sport, dénommés parfois sous le vocable de supporters périphériques (satellite fans) (Kerr et Gladden, 2008), soutenir un club ou un athlète qui évolue dans un autre pays ou un autre sport. De la même manière, de nombreuses femmes semblent exprimer une volonté de participer à des événements sportifs majeurs tels que les Jeux Olympiques ou les Coupes du Monde mais, dans ce cas précis, elles s'engageraient selon leurs propres mode et logique de consommation qui s'articuleraient autour de l'esthétisme, de la fête, de l'affect et de l'émotion (Lipovetsky, 1998).

Aujourd'hui, les formes de participation et de consommation de ces nouveaux publics se situent à différents niveaux et passent par toutes sortes d'identification : classe sociale, âge, genre, ethnicité, territoire local ou national...

Dans le même temps, le comportement des supporters plus traditionnels ne peut qu'être affecté par cette différenciation des publics et les nouvelles façons d'aborder le spectacle. Les nombreux contextes sociaux liés à la réception du spectacle sportif sont ainsi devenus des lieux de constructions et d'expressions identitaires plus complexes, 
dépassant les simples appartenances à des communautés territoriales. Les groupes de supporters deviennent des acteurs du spectacle à part entière plus ou moins organisés. Même si cette situation n'est pas nouvelle, elle semble avoir pris des proportions considérables dans certains pays européens pour des raisons économiques, géopolitiques, religieuses, culturelles, ethniques... Les conséquences sociales les plus visibles de ce phénomène sont sans doute l'émergence, voire la reconnaissance, de nouvelles formes de violence ritualisée (symbolique, verbale, physique) et de discrimination (de race, de territoire, d'ethnie, de genre...). Même si ces formes de déviance existent depuis longtemps chez les supporters de football (Bodin, 2003) qui se déplacent au stade pour soutenir et encourager leur équipe, elles semblent s'amplifier dans d'autres sports comme le basket ball ou le rugby (Bernache-Assolant, 2006) L'enjeu sociétal devient important et nous amène à essayer de comprendre ces nouvelles formes de supportérisme. Ainsi, après avoir souligné la polymorphie des comportements des spectateurs dans les enceintes sportives, nous proposerons un nouveau cadre d'analyse socio-psychologique des relations entre les groupes de spectateurs qui se rendent dans ces enceintes et qui peuvent potentiellement avoir des relations conflictuelles, voire violentes ou discriminatoires. Par la suite, ce cadre pourra être élargi à d'autres lieux et modes de consommation du spectacle sportif, et donc à de nouveaux publics.

\section{Les spectateurs dans une enceinte sportive : un objet d'étude polymorphe}

La consommation du spectacle sportif est un objet d'étude polymorphe. En effet, de nombreux paramètres peuvent faire varier les niveaux d'analyse des expériences vécues et des comportements des publics dans les enceintes sportives. Ces paramètres dépendent de l'objet lui-même en tant que produit culturel et « spectacle vivant ».

\section{Le spectacle sportif : produit culturel et spectacle vivant}

En tant que produit culturel, la consommation du spectacle sportif est intégrée et dépendante d'un univers socio-économique et symbolique à la fois lié aux référents culturels de la population et aux cultures sportives. Du point de vue des référents culturels, les nombreux travaux 
portant sur les différences interculturelles de consommation (Solomon, 2005) ont mis en évidence que les populations appartenant à des continents, des pays ou des régions dissemblables consommaient des produits culturels de manière hétérogène en fonction de leur niveau de développement, de leur histoire, de leur éducation... Ainsi, les rencontres de sports collectifs les plus importantes aux Etats-Unis sont liées au football américain, au base-ball, au basket-ball et au hockey sur glace, alors qu'en Europe le football, le rugby, voire le handball, sont prédominants. De plus, même si le sport se veut universel, son spectacle ne suscite pas les mêmes engouements en fonction des événements (Jeux Olympiques, Coupes du Monde, Championnats nationaux...) et de l'accessibilité aux retransmissions médiatiques (satellite, Internet, etc.). Du point de vue des cultures sportives, à chaque match correspondrait un type de public dépendant de la nature et du volume de son « capital culturel et économique ». En Europe, malgré des niveaux de développement sensiblement différents entre les populations des Etats membres, la fréquentation et le suivi des spectacles sportifs distingueraient les publics sauf peut être pour le football qui semble engendrer une adhésion plus large.

Un phénomène nouveau est venu se greffer sur la consommation du spectacle sportif : la médiatisation des publics en les inscrivant dans des cultures et des motivations plus vastes que celles du seul champ sportif. La retransmission de manifestations identitaires, individuelles ou collectives, dramatiques ou festives, fait aujourd'hui pleinement partie des éléments du spectacle sportif au sens où les compétiteurs ne sont pas les seuls acteurs de ces spectacles vivants. La mise en place de ces boucles de rétroaction, au sens où les comportements des joueurs et des spectateurs sont eux-mêmes conditionnés par leur médiatisation, favoriserait davantage les phénomènes de violence ou de discrimination, et seraient à l'origine des actions déviantes des «streakers » et autres voleurs d'images... Afin de faire rêver et de divertir, tout est bon pour alimenter ou pigmenter la retransmission du spectacle : gros plans sur les VIP ou des femmes au physique avantageux, zooms sur certaines parties anatomiques des joueurs et des spectateurs, etc. On est alors loin d'une conception où le sport est directement un vecteur de socialisation, d'apprentissage de la citoyenneté et un moyen d'insertion par les valeurs qu'il véhicule (respect de l'adversaire, effort...) à travers ses acteurs. Au contraire, dans nos sociétés surmédiatisées, il semble que le spectacle soit devenu un support de construction de groupes dont les enjeux dépassent largement la compétition sportive. 
La fréquentation, voire l'occupation, des enceintes sportives peut faire l'objet d'un véritable affrontement entre des groupes de supporters qui ne se préoccupent plus forcément du déroulement du jeu sur le terrain, mais tiennent avant tout à être visibles, à avoir une tribune médiatique. Les sifflets de la Marseillaise en France durant les matches de football (France-Tunisie en 2008) sont un autre type de manifestations où la politisation des tribunes prend le pas sur les fins sportives.

\section{L'étude des nouveaux publics : un objet d'étude complexe}

Les caractéristiques de la consommation de spectacle sportif semblent montrer que les spectateurs recherchent, simultanément ou alternativement, des expériences individuelles et interpersonnelles (contiguës ou partagées), sous forme médiatisée et/ou vécue sur site, avec de fortes dimensions hédoniques et symboliques (Bouchet et Pulh, 2006). L'expérience de consommation du spectateur étant créée à partir d'une interaction spécifique entre un dispositif de diffusion (audiovisuel, Internet, portable ; direct, différé, en continu) et les pratiques des individus dans un contexte donné (sur site ou hors site ; à domicile ou dans un espace public), il est possible de dégager quatre grandes formes de consommation du spectacle sportif et donc quatre catégories de spectateurs ( $c f$. Figure 1). Dans le cadre de cette recherche, nous nous focaliserons uniquement sur les spectateurs qui se déplacent dans un site, souvent un stade ou un palais des sports, pour assister à une rencontre.

Figure 1. Les quatre formes de consommation du spectacle sportif

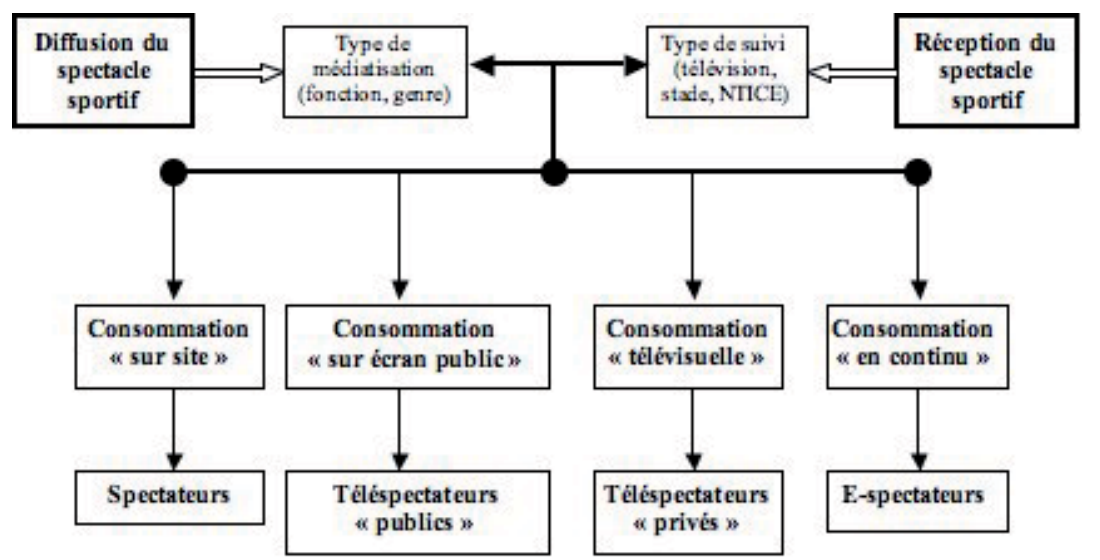


La littérature traitant des comportements des spectateurs de sport au stade est historiquement dominée par les travaux d'inspirations ethnologique, sociologique et économique en relation avec la violence, réelle ou symbolique, observée au sein des enceintes sportives. A ce titre, nous pouvons citer les travaux de Bodin (2003), Dunning (1999), Bromberger (1995) et Giulianotti, Bonney et Hepworth (1994) qui traitent du phénomène du hooliganisme. Dans une autre perspective, nous pouvons évoquer les approches psychosociales nord-américaines (e.g. Dietz-Uhler et Murrell, 1999 ; Wann, Tucker et Schrader, 1996) qui mettent l'accent sur le concept d'identification à l'équipe définie comme la propension qu'a un individu à se percevoir comme un supporter de l'équipe, à être impliqué vis-à-vis de cette équipe, à être concerné par les performances de l'équipe et à voir l'équipe comme une représentation de lui-même. Cette identification constituerait un antécédent majeur à de nombreuses réponses cognitives, affectives et conatives. Selon Wann et Branscombe (1993), l'identification à l'équipe est expliquée par l'attachement que les individus montrent pour leur équipe, l'argent qu'ils dépensent et le temps qu'ils consacrent pour la suivre. De manière plus précise, les individus faiblement identifiés semblent relativement désintéressés : ils ne portent pas d'accessoire ou de vêtement aux couleurs du club et n'applaudissent ou ne supportent pas leur équipe. Le rôle de supporter peut ainsi être considéré comme une composante simplement périphérique du concept de soi (Wann, Melnick, Russel et Pease, 2001). A l'opposé, les spectateurs fortement identifiés démontrent un engagement très important et ce rôle représente une composante essentielle de leur identité (Tajfel, 1981 ; Tajfel et Turner, 1986). Ils portent des vêtements aux couleurs de l'équipe, ils se maquillent, ils essayent de perturber ou d'influencer l'adversaire, ils s'opposent verbalement et parfois physiquement aux supporters de l'équipe adverse, ils s'approprient les victoires et les défaites de leur équipe (e.g. Mahony, Howard et Madrigal, 2000). Dans cette perspective, les travaux psychosociaux traitant des événements sportifs reconnaissent l'identification à l'équipe comme un modulateur d'intensité des comportements et des émotions des spectateurs, que ce soit avant, pendant ou après la rencontre. Dans cette recherche, nous centrerons notre analyse sur les relations intergroupes potentiellement violentes ou discriminatoires des spectateurs des enceintes sportives pour suivre une rencontre en direct. 


\section{L'analyse des relations intergroupes potentiellement violentes ou discriminatoires des spectateurs}

Le point de vue adopté est de considérer « le spectacle sportif» comme une situation de communication totale (Brown et Fraser, 1979) avec ses scènes et ses participants en interaction. Le spectacle dans les enceintes sportives diffère du point de vue « sportif» et «territorial». Les sports individuels versus sports collectifs, les affrontements directs (tennis) versus affrontements indirects (patinage artistique) et les enjeux compétitifs versus non compétitifs (show, démonstration) font varier le spectacle, tout comme les ancrages territoriaux. En effet, le spectacle sportif, en fonction de la nature du territoire d'appartenance des équipes ou des athlètes regardés : clubs ou sportifs locaux versus équipes ou sportifs nationaux versus équipes ou sportifs continentaux, ne renvoie pas aux mêmes enjeux. Cependant, pour l'étudier, nous proposons de nous centrer sur la situation sportive prototypique : le match opposant deux équipes ( $c f$. Figure 2). En effet, cette situation semble depuis l'antiquité (Piotrowsky, 2006) propice à générer de la violence et de la discrimination.

Figure 2. La scène du spectacle sportif dans le stade

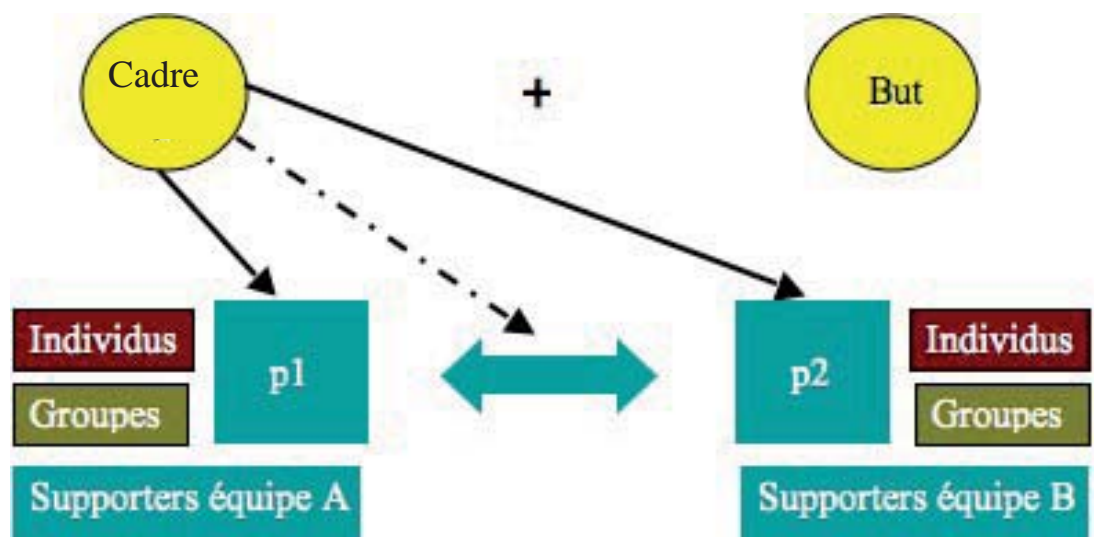

Pour nous, recourir à la situation de communication totale revient à inscrire les différents éléments impliqués dans la logique propre à la rencontre sportive. Cette situation peut faire appel à des scènes différentes. Celles qui sont liées à la fréquentation de lieux spécifiques dans le 
site de la rencontre : abord du match, buvette, vestiaire, couloir d'entrée, salle VIP..., avec des acteurs particuliers (forces de l'ordre, personnels de services, service technique...). Celles qui se déroulent en dehors du site : les abords proches (rues, commerces), les parkings... Néanmoins, la scène structurante est la scène du match, celle où des spectateurs placés sur les gradins regardent en direct s'affronter les équipes en espérant la victoire de celle qu'ils soutiennent. Dans cette scène, les acteurs du jeu (athlètes ou joueurs en action) représentent le cœur du spectacle dans le sens où sans eux l'événement n'aurait pas lieu d'être. Même si, à ceux-ci s'ajoutent d'autres acteurs de moindre visibilité, à savoir les staffs techniques (entraîneur, préparateur...) et les corps médical et réglementaire qui régulent le déroulement de la rencontre (arbitre, etc.), ils sont anecdotiques. Les seconds acteurs phare de la scène, ce sont les supporters. Ils ne correspondent qu'à une partie des publics sur site allant des supporters " périphériques » aux «fans traditionnels». Ce sont ces derniers qui supportent, sans ambiguïté, l'une des deux équipes et réagissent au déroulement du match. Fortement identifiés à leur équipe, ils sont pour la plupart abonnés, ils font souvent partie d'un club et arborent des accessoires (casquette, maillot, écharpe, autocollant sur les voitures, etc.) qui les rendent visibles pour les autres spectateurs. Ainsi, ils constituent avec les joueurs le cour de la scène principale du spectacle sportif en direct, ce qu'entérinent, lors des retransmissions, les médiateurs de l'événement (cameraman, photographe...). Pour ces supporters hautement identifiés, souvent qualifiés d'ultra, les résultats des matchs correspondent à des enjeux identitaires qui les poussent à réagir, parfois même hors du stade, lorsque leur équipe perd. Ils peuvent entraîner des mouvements d'adhésion des publics plus périphériques et constituent les éléments clés des débordements possibles en termes de violence et de discrimination.

Le cadre : les paramètres spatio-temporels ont des incidences sur les participants. En plus de la chaleur comme facteur favorisant la violence (voir Russel, 2004), sont actuellement pris en compte le bruit, la densité du public et la position debout/assise. Ces derniers éléments situationnels semblent jouer sinon directement sur les comportements agressifs du moins sur l'excitation physiologique des spectateurs qui deviendraient plus disposés à réagir. De plus, des éléments plus socialisés, tel l'anonymat lié au grand nombre de spectateurs (Wann, Haynes, Mclean et Pullen, 2003) et l'obscurité, ainsi que l'autorisation implicite de l'alcool, semblent favoriser la production d'actions antisociales, même si certains sports sont davantage porteurs de normes permissives 
vis-à-vis de la violence que d'autres (Mustonen, Arms et Russel, 1996). Ainsi, certains éléments du cadre spatio-temporel favoriseraient, via l'excitation, des conduites expressives qui pourraient devenir violentes par désinhibition de certains interdits. La présence d'observateurs, par la médiatisation du match, est, de ce point de vue, intéressante.

Traditionnellement, les observateurs présents ou distants modifient les conduites. Là encore, le sens de la variation dépend des situations. Il peut se produire un emballement des conduites lorsque les sujets se sentent l'objet d'une attention particulière (« Hawthone effect »). Les individus de faible statut, mais relativement investis dans un rôle, peuvent tenter de montrer leurs capacités, généralement sous-estimées, lorsqu'on s'intéresse à eux. Ainsi, des supporters en voie d'intégration dans leur groupe peuvent manifester, parce qu'ils sont filmés, ce qui a par ailleurs été nommé un effet PIP (Codol, 1984). Ils vont donner à voir le comportement prototypique de leur groupe et, en ce sens, être les caricatures de ce groupe. Lorsqu'ils sont moins bien reconnus que d'autres, ils peuvent vivre le soutien à leur équipe comme un challenge et essayer de dépasser les supporters plus installés. Dans le stade, comme précédemment mentionné, le rapport à la norme dépend du type de pratiques, certaines étant associées à la violence des supporters. Dans ce cas, notamment pour le football, la violence ou le racisme, traits stéréotypiques les plus spectaculaires, deviennent particulièrement disponibles. La télévision, en rendant publique l'appartenance du spectateur filmé au groupe des supporters, constitue un facteur d'accentuation de cette mobilisation identitaire et peut ainsi induire le recours à des comportements agressifs, violents ou discriminatoires.

Les buts : si les buts sociétaux (divertir) et sociaux (participer à l'événement) restent stables, les évolutions de la situation de jeu (victoire ou défaite des équipes) font varier les buts situationnels : la scène pourra alors s'orienter sur le maintien d'un statut quo, sur la stabilisation des acquis de l'un des groupes ou enfin sur le renversement de la situation. La saillance des enjeux liés aux scores et à l'ancrage du match dans les niveaux territoriaux rend la scène plus ou moins explosive. En effet, plus les scores sont serrés, plus les possibles sont ouverts, plus l'éveil psychophysiologique est fort. Un affrontement violent entre les joueurs sur le terrain peut se répercuter sous forme d'un conflit chez les spectateurs (Arms, Russel et Sandilands, 1979). De la même façon, une décision d'arbitrage contestable peut déclencher des débordements.

Ainsi, la scène, indépendamment des participants et de leurs enjeux, contribue à l'émergence de la violence. Le cadre, proprement 
dit, avec ses coordonnées spatio-temporelles favorisant l'excitation et la désinhibition des interdits, mais aussi la médiatisation de l'événement qui renforce l'appropriation des appartenances groupales, constitue un environnement facilitant de conduites extrêmes. Les buts situationnels, en connexion avec le déroulement de la partie, potentialisent le climat précédemment institué en rendant sensible aux événements négatifs, telles les altercations sur le terrain, les erreurs d'arbitrage. Cependant, la prise en compte des particularités des participants est nécessaire pour rendre compte des passages à l'acte et des différentes formes qu'ils peuvent prendre.

Les participants : les supporters hautement identifiés, bien qu'ils ne soient pas les seuls participants du spectacle sportif in situ, y jouent un rôle déterminant. En effet, ils adoptent, en mobilisant leur identité sociale, des conduites collectives qui peuvent être suivies dans certaines circonstances par des spectateurs moins engagés. L'identité sociale distincte de l'identité personnelle est l'identité adoptée par les sujets qui se considèrent en tant que membres de groupes. Elle est d'autant plus investie par le sujet qu'il entretient des liens forts à son groupe d'appartenance. En ce sens, les supporters hautement identifiés mobilisent nécessairement leur identité sociale lors des matchs et se comportent en fonction de cette dernière. Selon Tajfel (1978), l'appartenance à un groupe opposé à un autre groupe suffit à produire des effets discriminants vis-à-vis du groupe dont on ne fait pas partie. Les membres de l'autre groupe sont, via les stéréotypes, perçus comme identiques (Castel, 1999) ; ils sont automatiquement jugés plus négativement que soi et sont les cibles désignées de comportements discriminants. De ce fait, les supporters d'une équipe sont a priori tout disposés à activer ces mécanismes vis-à-vis des supporters de l'équipe adverse.

Ces effets génériques sous tendent les relations des supporters hautement identifiés d'équipes adverses indépendamment du match proprement dit. Le dénigrement de l'autre équipe, labellisé «Blast» depuis l'étude princeps de Cialdini et Richardson (1980), opère quels que soient les résultats de l'équipe adverse (End, 2001 ; Wann et Grieve, 2005). Quant aux attaques effectives envers l'autre groupe, pour les opposants dits héréditaires, tels le Paris St Germain et Marseille en France ou Manchester United et Arsenal en Angleterre, elles peuvent avoir lieu à l'extérieur du stade et indépendamment de ce qui s'y joue, la médiatisation du match annoncé réactualisant, rendant plus disponible la catégorisation existante, ses effets automatiques et les formes, 
dont la violence, qu'ils ont déjà prises. Ces formes semblent dépendre des partitions structurant les groupes de supporters entre eux et de leur incidence en termes de remaniement identitaire. En effet, dans l'enceinte du stade, les aléas des scores jouent sur l'identité des sujets hautement identifiés. Lorsque l'équipe supportée gagne, l'estime de soi des membres du groupe est forte ; par contre, lorsque l'équipe perd, elle remet en cause la valeur des sujets qui ont cru en elle et les oblige à mettre en place des stratégies de « management identitaire ». Or, il apparaît que ces stratégies diffèrent selon la structuration des différents groupes de supporters.

\section{Les différents groupes de supporters hautement identifiés}

Dans le cadre du supportérisme, le lien au groupe est dépendant du type de rapport que les membres entretiennent avec l'équipe. Certains groupes, attachés au territoire ou à certaines valeurs, trouvent dans le soutien à leur équipe un moyen de les faire valoir. Au fil des matchs, en s'opposant à d'autres qui soutiennent les équipes adverses, ils testent et renforcent ces liens. Les groupes supportant une équipe parce qu'ils sont fiers d'appartenir à une ville, une région ou un pays pour ce qu'il est ou ce qu'il représente, vont se retrouver face à d'autres groupes de supporters animés comme eux par le même type d'enjeux. La confrontation des deux parties dans le stade relève d'une partition oppositive facilitant la mise en place de conduites ciblées peu amènes pour 1 '« ennemi du jour».

Cependant, ce mode de structuration des groupes n'est pas le seul possible. Des sujets particuliers peuvent s'affilier pour constituer un groupe qui pourra se distinguer de la majorité. Dans le cadre du supportérisme, certaines caractéristiques individuelles renvoyant à ce que Brown et Fraser (1979) appellent l'« individu en tant qu'individu », peuvent précéder l'affiliation groupale. L'agressivité, relevée comme trait de caractère, a pu très tôt produire le rejet par leurs pairs des individus ayant adopté des comportements violents. Lorsque les relations interindividuelles sont difficiles, les individus ne peuvent avoir une identité personnelle favorable et se trouvent très tôt relégués dans des groupes considérés par les autres comme antisociaux (Amodeï et Scoot, 2002). Cependant, les sujets qui y appartiennent ne peuvent se considérer comme tels. Recherchant comme tout un chacun une estime de soi favorable, ils peuvent soit valoriser le trait support de leur relégation, soit rechercher ailleurs une reconnaissance identitaire. Le suppor- 
térisme est sans doute de ce point de vue une affiliation prisée. Le sport, comme nous l'avons précédemment évoqué, est devenu une pratique sociale particulièrement valorisée. Les joueurs, par la médiatisation des matchs, sont des idoles qui peuvent permettre d'acquérir un peu de leur gloire à ceux qui les approchent. Le BIRG (Basking In Reflected Glory) est en effet un mécanisme repéré par Cialdini, Borden, Walker, Freeman et Sloan (1976) qui consiste à se rapprocher de personnes ou de groupes socialement valorisés pour atteindre soi-même un peu de renommée. De plus, le spectacle sportif dans son extension peut même rendre célèbres les supporters pour eux-mêmes. Si leur animation est bonne, ils peuvent obtenir une reconnaissance directe. En conséquence, devenir supporter peut revenir à adopter, pour ceux qui sont considérés comme des jeunes célibataires économiquement pauvres et socialement désavantagés (Mustonem, Arms et Russel, 1996), une identité sociale enviable. L'entrée dans le supportérisme se faisant, via le BIRG, par les joueurs, les actions des supporters suivent les résultats des équipes, les succès et défaites lors des matchs devenant déterminants. Si l'équipe gagne, ils sont forts, si l'équipe perd, ils sont faibles.

Autour des équipes de joueurs gravitent donc des groupes de supporters qui ne renvoient pas nécessairement à des groupes de même nature. Il s'ensuit que les modes de régulations identitaires face aux succès et aux défaites de l'équipe ne sont pas identiques, amenant ainsi les supporters à réagir de façon différente aux résultats des matchs.

\section{Place de la discrimination et de la violence dans les modes de régulation identitaires des groupes de supporters}

Dans les groupes oppositifs, le dénigrement de l' autre groupe existe toujours, les insultes peuvent fleurir et, s'il est possible de produire des effets négatifs, ils seront produits. Lors de l'échec, les supporters se désolidarisent de l'équipe mais restent des supporters. Partageant un même sort, ils s'associent à tous ceux qui soutiennent la même équipe (Bernache-Assollant, Lacassagne et Braddock, 2007). Certes, l'équipe a perdu mais eux, les supporters, ont fait leur travail. En d' autres termes, pour réguler leur estime d'eux-mêmes, les supporters des groupes oppositifs opèrent une re-catégorisation plus favorable. Ils n'auraient pas a priori besoin d'aller au-delà pour se sentir bien et la discrimination ou la violence ne paraît pas une nécessité. Pour ces groupes, le cadre et les buts situationnels sont déterminants. La discrimination ou la violence peut être exacerbée par les éléments contextuels. Plus parti- 
culièrement, les groupes oppositifs étant fondés sur la valeur (Castel et Lacassagne, 2005), ils devraient être sensibles au Sentiment d'Injustice Subie (SIS) et ce d'autant plus que l'issue de la rencontre est incertaine. Selon Lerner (1980), les individus ont besoin de croire qu'ils vivent dans un monde juste où chacun a ce qu'il mérite et mérite ce qu'il a. Si cette croyance est menacée par l'observation ou le vécu d'injustices, les individus sont en mesure de restaurer par l'action leur croyance en un monde juste. Le SIS devient donc un affect susceptible d'engendrer de la violence (Goutas, Girandola et Minary, 2003), particulièrement quand les supporters sont psychophysiologiquement excités. Les groupes oppositifs, à défaut de pouvoir s'en prendre directement aux arbitres ou aux joueurs sources de l'injustice mais dotés d'un haut statut qui les rend intouchables (Gilles et Clémence, 2007), pourraient vouloir en découdre avec ceux de l'autre groupe, supporters et joueurs, pour montrer qu'ils sont les plus forts. En conclusion, pour ces supporters hautement identifiés, engagés dans une relation de type oppositif, les éléments du cadre sont les déterminants dans la violence de leurs réactions.

Pour les groupes minoritaires, par contre, le rôle de l'environnement semble différent. Lorsque l'équipe perd, la minorité hautement identifiée s'en désolidarise et se replie sur elle-même. Si les groupes sont structurés autour de la possession du trait « discriminant» ou «violent » que les autres, à savoir la majorité, ne partagent pas, alors le recentrage sur les standards de la catégorie ne peut qu'exhumer ce trait. Les supporters qui se sont fédérés autour d'une équipe pour acquérir une image valorisée se retrouvent, en cas de défaite, dans la partition fondatrice. Ils ne sont plus les supporters de l'équipe $\mathrm{X}$, mais les « racistes » ou les « violents » opposés à l'ensemble de la population qui ne possède pas ce trait. Pour exister, les « violents » n'ont plus qu'à valoriser leur spécificité et donc déployer leur force sur tous ceux qui n'appartiennent pas à leur communauté. Etant par constitution l'élite de la violence, ils ne peuvent que gagner et donc, aller dans l'action jusqu'à l'affirmation incontestable de leur pouvoir. Pour eux, le cadre ne peut suffire à réguler l'atteinte à l'estime de soi produite par l'échec de l'équipe. Ils doivent, par eux-mêmes, retrouver leur honneur et donc se battre et vaincre. Dans ce contexte, la médiatisation du spectacle peut renforcer les comportements asociaux. La mise en exergue de la réaction des Ultra violents à la suite de la défaite de leur équipe ne peut que favoriser l'identification au nouveau groupe émergent. 
Ainsi, les groupes de supporters n'entretiennent pas le même type de rapport à l'agression selon la structure groupale dans laquelle ils s'inscrivent. Les groupes relevant d'une partition oppositive pourront manager leur identité menacée sans recours à la discrimination ou la violence, sauf s'ils sont encouragés par le cadre spatio-temporel, la présence d'observateurs pouvant constituer un frein aux débordements. A l'inverse, les groupes relevant d'une partition minoritaire/majoritaire fondée sur le regroupement de personnes a priori agressives ou conflictuelles qui ont pu trouver, via le supportérisme, une identité sociale favorable, seront prêts, lorsque cette identité est menacée par l'échec de leur équipe, à adopter des comportements déviants sans cible précise. Le cadre spatio-temporel aura peu de chance de canaliser leur violence qui pourrait exister sans l'état d'excitation physiologique particulier produit par le cadre ou le but de la rencontre. Par contre, la présence de média, attestant de leur professionnalisme dans la déviance, pourra favoriser son développement.

\section{Conclusion}

L'analyse du match à travers le modèle de Brown et Fraser (1979) permet ainsi de mieux comprendre les déterminants des conduites agressives dans le stade au moment de l'événement sportif. Non seulement ce modèle aide à repérer les facteurs contextuels susceptibles de favoriser par eux-mêmes des comportements conflictuels violents ou discriminatoires, mais il permet surtout de prendre en compte le rôle déterminant des relations entre participants. Le match en tant que scène inscrit ces dernières dans la perspective des conflits intergroupes. Le développement du spectacle sportif ne peut que renforcer le caractère spectaculaire de l'affrontement. En donnant une place accrue aux différents acteurs, et notamment aux supporters, la médiatisation des événements favorise l'émergence de conduites à risque. Pour les supporters qui sont engagés dans une partition oppositive, par exemple par attachement territorial, la mise en scène du spectacle favorise l'excitation généralisée et rend plus probable des affrontements pour défendre son camp contre l'autre.

Pour les supporters fédérés autour de la violence et formant une minorité qui se distingue de la majorité qui ne possède pas ce trait, un mauvais score se traduira par un repli sur ses valeurs premières et pourra déboucher sur la violence tous azimuts, les minorités visibles 
constituant des cibles privilégiées. Les fans semblant préférer les sportifs relevant de la même appartenance qu'eux (Burdekin, Hossfeld, Smith, 2005), lorsque la violence est dirigée vers les joueurs, ceux qui n'appartiennent pas à leur groupe ethnique sont les premiers visés. De fait, les joueurs de couleur sont plus agressés dans leur propre camp que les joueurs du camp adverse (Holland, 1995), mais aussi les quelques spectateurs de couleurs et, par effet de halo, les minorités ethniques habitant près des stades (Long, Carrington et Spacklen, 1997). On comprend alors mieux les manifestations de racisme vis-à-vis des Noirs dans le football français ou italien, qu'elles concernent les joueurs ou les spectateurs.

\section{Références bibliographiques}

Amodeï, N., Scott, A. A. (2002). Psychologists' contribution to the prevention of youth violence. The Social Science Journal, 39, 511-526.

Arms, R. L., Russell, G. W., \& Sandilands,M. L. (1979). Effects of viewing aggressive sports on the hostility of spectators. Social Psychology Quarterly, 42, 275-279.

Bernache-Assollant, I. (2006). Contextes intergoupes et stratégies de gestion identitaire chez les supporters. Thèse de Doctorat de l'Université de Bourgogne, Dijon, France.

Bernache-Assollant, I., Lacassagne, M.-F., \& Braddock II, J.H. (2007). Basking in Reflected Glory and Blasting: Differences in identity-management strategies between two groups of highly identified soccer fans. Journal of Language and Social Psychology, 26, 381-388.

Bodin, D. (2003). Le hooliganisme. Paris: PUF.

Bouchet, P., \& Pulh, M. (2006). L'expérience vécue : un autre regard sur l'analyse du comportement du consommateur. $5^{\text {Emes }}$ Journées Normandes de recherche sur la consommation, Caen, FR.

Bromberger, C. (1995). Le match de football. Ethnologie d'une passion partisane à Marseille, Naples et Turin. Paris: Maison des Sciences de l'Homme.

Brown, P., Fraser, C. (1979). Speech as a marker of situation. In R. K. Scherer, H. Giles (Eds.), Social markers in speech. Cambridge, Cambridge University Press, 33-63.

Burdekin, R. C. K., Hossfeld, R.T., Smith, J.K. (2005). Are NBA Fans Becoming Indifferent to race? Journal of sports economics, 2(6), 144-159.

Castel, P. (1999). Discrimination et langage. In J.-P. Pétard (Ed.), Psychologie Sociale. Paris, Bréal Editions, 317-374.

Castel, P., Lacassagne, M.-F. (2005). Les partitions discriminantes dans la négociation du contrat de communication. Bulletin de Psychologie, 58 (3), 299-306.

Cialdini, R. B., Bordon, R. J., Thorne, A., Walker, M. R., Freeman, S., Sloan, L. R. 
(1976). Basking in reflected glory: Three field studies. Journal of Personality and Social Psychology, 34, 366-375.

Cialdini, R. B. \& Richardson, K. D. (1980). Two indirect tactics of image management: Basking and blasting. Journal of Personality and Social Psychology, 39, 406-415.

Codol, J.P. (1984). Semblables et différents : recherches sur la quête de la similitude et de la différence. Lille 3 : Atelier national de reproduction des thèses.

Dietz-Uhler, B., \& Murrell, A. (1999). Examining fan reactions to game outcomes: A longitudinal study of social identity. Journal of Sport Behavior, 22, 15-27.

Dunning, E. (1999). Sport matters: Sociological studies of sport, violence and civilization. London: Routledge.

End, C. M. (2001). An examination of NFL, fans' computer mediated BIRGing. Journal of Sport Behavior, 24 (2), 162-181.

Gilles, I., \& Clémence, A. (2007), Attribution d'intentions hostiles et intentions comportementales agressives dans un contexte intergroupe. Revue Internationale de Psychologie Sociale, 20(4), 63-88.

Giulianotti, R., Bonney, N., \& Hepworth, M. (1994). Football, violence and social identity. London: Routledge.

Goutas, N., Girandola, F., Minary, J. P. (2003). Le Sentiment d'Injustice Subie : un nouveau regard sur l'agression. Revue Internationale de Psychologie Sociale, 16, 125-149.

Holland, B. (1995). Kicking racism out of football: an assessment of racial harassment in and around football grounds, New community, 21 (4), 567-86.

Kerr, A. K., \& Gladden, J. M. (2008). Extending the understanding of professional team brand equity to the global marketplace. International Journal of Sport Management and Marketing, 3(1/2), 58-77.

Lerner, M. J. (1980). The Belief in a just world. A fundamental delusion. New York: Plenum Press.

Lipovetsky, G. (1998, 14/7/1998). L'engouement féminin. Libération.

Long, J., Carrington, B., \& Spracklen, K., (1997). Asians cannot wear turbans in the scrum: explorations of racist discourse within professional rugby league'. Leisure Studies, 16(4), 249-260.

Mahony, D. F., Howard, D. R., \& Madrigal, R. (2000). BIRGing and CORFing behaviors by sport spectators: High self-monitors versus low self-monitors. International Sports Journal, 4(1), 87-106.

Mustonen, A., Arms, R. L., Russel, G. W. (1996). Predictors of sports spectators' proclivity for riotous behaviour in Finland and Canada. Person. Individ. Diff., 21 (4), 519-525.

Piotrowski, P. (2006). Coping with football-related hooliganism: healing symptoms versus causes prevention, Journal of applied social psychology, 36, 3, 629-643.

Russel, G. W. (2004). Sportriots: A social-psychological review. Aggression and violent behaviour.

Solomon, M. R. (2005). Comportement du consommateur. Prentice Hall, Pearson Education Inc.

Tajfel, H. (1978). Differentiation between social groups: Studies in the social psychology of intergroup relations. London, Academic Press.

Tajfel, H. (1981). Human groups and social categories. Cambridge, UK: Cambridge University Press.

Tajfel, H., \& Turner, J. C. (1986). The social identity of intergroup behaviour. In S. 
Worchel \& W. G. Austin (Eds.), The psychology of intergroup relations (pp. 7-24). Chicago, MI: Nelson Hall.

Wann, D. L., \& Branscombe, N. R. (1993). Sports fans: Measuring degree of identification with the team. International Journal of Sport Psychology, 24, 1-17.

Wann, D. L., Grieve, F. G. (2005). Biaised evaluations of in-group and out-group spectator behavior at sporting events: the importance of team identification and threats to social identity, The Journal of Social Psychology, 145, 531-545.

Wann, D. L., Haynes, G., Mclean, B., Pullen, P. (2003). Sport team identification and willingness to consider anonymous acts and hostile aggression, Aggressive Behavior, $29,406-413$

Wann, D. L., Melnick, M., Russel, G., \& Pease, D. (2001). Sports fans: The psychology and social impact of spectators. New-York (NY): Routledge.

Wann, D. L., Tucker, K. B., \& Schrader, M. P. (1996). An exploratory examination of the factors influencing the origination, continuation, and cessation of identification with sports teams. Perceptual and Motor Skills, 85, 995-1001. 
